\title{
Blow-up criterion for 3D compressible viscous magneto-micropolar fluids with initial vacuum
}

\section{Peixin Zhang*}

\section{"Correspondence:} zhpx@hqu.edu.cn School of Mathematical Sciences, Huaqiao University, Quanzhou, 362021, P.R. China

\begin{abstract}
In this paper, the author establishes a blow-up criterion of strong solutions to 3D compressible viscous magneto-micropolar fluids. It is shown that if the density and the velocity satisfy $\|\rho\|_{L^{\infty}\left(0, T ; L^{\infty}\right)}+\|u\|_{L^{s}\left(O_{1}, T_{i} L^{r}\right)}<\infty$, where $\frac{2}{s}+\frac{3}{r} \leq 1$ and $3<r \leq \infty$, then the strong solutions to the Cauchy problem can exist globally over $\mathbb{R}^{3} \times[0, T]$. The initial density may vanish on open sets, that is, the initial vacuum is allowed.
\end{abstract}

MSC: 76N10; 35B44; 35B45

Keywords: compressible magneto-micropolar fluids; blow-up criterion; strong solution; vacuum

\section{Introduction}

In this paper, we consider the following 3D compressible viscous magneto-micropolar fluids:

$$
\left\{\begin{array}{l}
\rho_{t}+\operatorname{div}(\rho u)=0, \\
(\rho u)_{t}+\operatorname{div}(\rho u \otimes u)-(\mu+\xi) \Delta u-(\mu+\lambda-\xi) \nabla \operatorname{div} u+\nabla P \\
\quad=2 \xi \nabla \times w+(\nabla \times H) \times H, \\
(\rho w)_{t}+\operatorname{div}(\rho u \otimes w)-\mu^{\prime} \Delta w-\left(\mu^{\prime}+\lambda^{\prime}\right) \nabla \operatorname{div} w+4 \xi w=2 \xi \nabla \times u, \\
H_{t}-\nabla \times(u \times H)=-\nabla \times(\sigma \nabla \times H), \\
\operatorname{div} H=0,
\end{array}\right.
$$

where $x=\left(x_{1}, x_{2}, x_{3}\right) \in \mathbb{R}^{3}$ is the spacial coordinate and $t \geq 0$ is the time. The unknown functions $\rho=\rho(t, x), u=u(t, x)=\left(u^{1}, u^{2}, u^{3}\right)(t, x), w=w(t, x)=\left(w^{1}, w^{2}, w^{3}\right)(t, x)$, $H=H(t, x)=\left(H^{1}, H^{2}, H^{3}\right)(t, x)$ and $P(\rho)=A \rho^{\gamma}(A>0, \gamma>1)$ are the fluid density, velocity, micro-rotational velocity, magnetic field and pressure, respectively. The constants $\mu, \lambda, \xi$, $\mu^{\prime}, \lambda^{\prime}$ and $\sigma$ are the viscosity coefficients of the fluid satisfying

$$
\mu, \mu^{\prime}, \xi, \sigma>0, \quad 2 \mu+3 \lambda-4 \xi \geq 0, \quad \text { and } \quad 2 \mu^{\prime}+3 \lambda^{\prime} \geq 0
$$

System (1.1)-(1.2) describing the motion of aggregates of small solid ferromagnetic particles relative to viscous magnetic fluids, such as water, hydrocarbon, ester, fluorocarbon, etc., in which they are immersed, covers a wide range of heat and mass transfer phe-

\section{Springer}

(c) 2013 Zhang; licensee Springer. This is an Open Access article distributed under the terms of the Creative Commons Attribution License (http://creativecommons.org/licenses/by/2.0), which permits unrestricted use, distribution, and reproduction in any medium, provided the original work is properly cited. 
nomena, under the action of magnetic fields, and is of great importance in practical and mathematics applications (see [1]). Indeed, (1.1) is composed of the balance laws of mass, momentum, moment of momentum and magnetohydrodynamic, respectively. Due to its importance in mathematics and physics, there is a lot of literature devoted to the mathematical theory of the compressible viscous magneto-micropolar system (see [2-4]).

For the incompressible magneto-micropolar fluid models where $\rho=$ Const., RojasMedar [5] established local existence and uniqueness of strong solutions by the Galerkin method. Ortega-Torres and Rojas-Medar [6] proved global existence of strong solutions for small initial data. A BKM type blow-up criterion for smooth solution that relies on the vorticity of velocity only was obtained by Yuan [7]. For regularity results, refer to Yuan [8] and Gala [9].

In particular, if the effect of angular velocity field of the particle's rotation is omitted, i.e., $w=0$, then (1.1) reduces to compressible magnetohydrodynamic equations (MHD). There are numerous important progress on compressible MHD (see [10-12] and the references therein). The local strong solutions to the compressible MHD with large initial data were respectively obtained by Vol'pert-Khudiaev [10] and Fan-Yu [11] in cases that the initial density is strictly positive and the initial density may vanish. Xu-Zhang [12] proved a blowup criterion that if $T^{*}<\infty$ is the maximal time of existence of a strong solution, then

$$
\sup _{T \rightarrow T^{*}}\left(\|\rho\|_{L^{\infty}\left(0, T ; L^{\infty}\right)}+\|u\|_{L^{s}\left(0, T ; L_{w}^{r}\right)}\right)=\infty
$$

where $L_{w}^{r}$ is the weak $L^{r}$ space and $r, s$ satisfy

$$
\frac{2}{s}+\frac{3}{r} \leq 1, \quad 3<r \leq \infty
$$

If $H=0$, (1.1) reduces to compressible micropolar fluid equations. Mujakovic [13, 14] considered the one-dimensional motion of compressible viscous micropolar fluids and studied the local/global existence. The global existence of strong solutions to the 1D model with initial vacuum was also obtained in [15]. For multi-dimensional compressible magneto-micropolar equations, Amirat and Hamdache [16] proved the global existence of weak solutions with finite energy and the adiabatic constant for $\gamma>3 / 2$, which generalized Lions' pioneering work [17] and the work by Feireisl et al. [18]. Chen [19] established the local existence and uniqueness of strong solutions under the assumption that the initial density may vanish, and in [20] Chen et al. proved a blow-up criterion that

$$
\sup _{T \rightarrow T^{*}}\left(\|\rho\|_{L^{\infty}\left(0, T ; L^{\infty}\right)}+\|\sqrt{\rho} u\|_{L^{s}\left(0, T ; L^{r}\right)}\right)=\infty,
$$

where $r, s$ satisfy (1.3).

If $H=0$ and $w=0,(1.1)$ reduces to isentropic compressible Navier-Stokes equations. In [21], the authors established a Serrin-type blow-up criterion that

$$
\sup _{T \rightarrow T^{*}}\left(\|\operatorname{div} u\|_{L^{1}\left(0, T ; L^{\infty}\right)}+\|\sqrt{\rho} u\|_{L^{s}\left(0, T ; L^{r}\right)}\right)=\infty,
$$

or

$$
\sup _{T \rightarrow T^{*}}\left(\|\rho\|_{L^{\infty}\left(0, T ; L^{\infty}\right)}+\|\sqrt{\rho} u\|_{L^{s}\left(0, T ; L^{r}\right)}\right)=\infty,
$$

where $r, s$ satisfy (1.3). 
In this paper, our main purpose is to establish a blow-up criterion of strong solutions for system (1.1) with the following conditions:

$$
\left\{\begin{array}{l}
(\rho, u, w, H)(x, 0)=\left(\rho_{0}, u_{0}, w_{0}, H_{0}\right)(x) \quad \text { in } \mathbb{R}^{3} \\
(\rho, u, w, H)(x, t) \rightarrow 0 \quad \text { as }|x| \rightarrow \infty
\end{array}\right.
$$

To proceed, we introduce the following notations. For $1 \leq r \leq \infty$, we denote the standard homogeneous and inhomogeneous Sobolev spaces as follows:

$$
\left\{\begin{array}{l}
L^{r}=L^{r}\left(\mathbb{R}^{3}\right), \quad D^{k, r}=\left\{u \in L_{\mathrm{loc}}^{1}\left(\mathbb{R}^{3}\right) \mid\left\|\nabla^{k} u\right\|_{\left.L^{r}<\infty\right\},} \quad\|u\|_{D^{k, r}}:=\left\|\nabla^{k} u\right\|_{L^{r}},\right. \\
W^{k, r}=L^{r} \cap D^{k, r}, \quad H^{k}=W^{k, 2}, \quad D^{k}=D^{k, 2}, \quad D^{1}=\left\{u \in L^{6} \mid\|\nabla u\|_{L^{2}}<\infty\right\} .
\end{array}\right.
$$

To present the main result, we first give the following local existence and uniqueness of strong solutions to the Cauchy problem (1.1), (1.2) and (1.4) with initial vacuum (without proof), which can be obtained by the same method developed by Choe-Kim in [22] (see also Fan-Yu [11] and Chen [19] for MHD and compressible micropolar fluids, respectively).

Theorem 1.1 Assume that for some $q \in(3,6]$, the initial data $\left(\rho_{0}, u_{0}, w_{0}, H_{0}\right)$ satisfy

$$
\begin{aligned}
& 0 \leq \rho_{0} \in L^{1} \cap H^{1} \cap W^{1, q}, \quad u_{0} \in D^{1} \cap D^{2}, \\
& w_{0} \in H^{2}, \quad H_{0} \in H^{2}, \quad \operatorname{div} H_{0}=0,
\end{aligned}
$$

and the compatibility conditions

$$
\begin{aligned}
& -(\mu+\xi) \triangle u_{0}-(\mu+\lambda-\xi) \nabla \operatorname{div} u_{0}+\nabla P_{0}-2 \xi \nabla \times w_{0}-\left(\nabla \times H_{0}\right) \times H_{0} \\
& \quad=\rho_{0}^{1 / 2} g_{1}, \\
& -\mu^{\prime} \triangle w_{0}-\left(\mu^{\prime}+\lambda^{\prime}\right) \nabla \operatorname{div} w_{0}-2 \xi \nabla \times u_{0}+4 \xi w_{0}=\rho_{0}^{1 / 2} g_{2},
\end{aligned}
$$

with some $\left(g_{1}, g_{2}\right) \in L^{2}$. Then there exists a positive time $T_{*} \in(0, \infty)$ such that the problem (1.1), (1.2) and (1.4) has a unique strong solution $(\rho, u, w, H)$ in $\mathbb{R}^{3} \times\left[0, T_{*}\right]$ satisfying, for some $q_{0} \in(3,6]$,

$$
\left\{\begin{array}{l}
\rho \in C\left(\left[0, T_{*}\right] ; L^{1} \cap H^{1} \cap W^{1, q_{0}}\right), \quad \rho_{t} \in L^{\infty}\left(0, T_{*} ; L^{2} \cap L^{q_{0}}\right), \quad \rho \geq 0, \\
(u, w, H) \in C\left(\left[0, T_{*}\right] ; D^{1} \cap D^{2}\right) \cap L^{2}\left(0, T_{*} ; D^{2, q_{0}}\right), \quad w \in C\left(\left[0, T_{*}\right] ; L^{2}\right), \\
H \in C\left(\left[0, T_{*}\right] ; H^{2}\right), \quad\left(\sqrt{\rho} u_{t}, \sqrt{\rho} w_{t}, H_{t}\right) \in L^{\infty}\left(0, T_{*} ; L^{2}\right), \\
\left(u_{t}, w_{t}, H_{t}\right) \in L^{2}\left(0, T_{*} ; D^{1}\right) .
\end{array}\right.
$$

Motivated by [20,21] and [12], we have the main purpose in this paper to prove a blowup criterion for the problem (1.1), (1.2) and (1.4). More precisely, the main result in this paper reads as follows.

Theorem 1.2 Assume that the initial data $\left(\rho_{0}, u_{0}, w_{0}, H_{0}\right)$ satisfies (1.5)-(1.7). Let ( $\rho, u$, $w, H)$ be a strong solution of the Cauchy problem (1.1), (1.2) and (1.4) with the regulari- 
ties (1.8). If $T^{*} \in(0,+\infty)$ is the maximal time of existence, then

$$
\lim _{T \rightarrow T^{*}}\left(\|\rho\|_{L^{\infty}\left(0, T ; L^{\infty}\right)}+\|u\|_{L^{s}\left(0, T ; L^{r}\right)}\right)=\infty
$$

for any $r$ and s satisfying (1.3).

Remark 1.3 Theorem 1.1 proves that the strong solutions of (1.1), (1.2) and (1.4) can exist only in a small time $T_{*}$, which means that if $T_{*}$ is the maximal time of existence, then there must be some component of the fluid mechanics blow-ups. Theorem 1.2 points out one kind of blow-up mechanics.

Remark 1.4 There is no any additional growth condition on the micro-rotational velocity $w$ and magnetic field $H$. This reveals that the density and the linear velocity play a more important role compared to the angular velocity of rotation of particles and the magnetic field in the regularity theory of solutions to 3D compressible magneto-micropolar fluid flows.

The rest of the paper is devoted to completing the proof of Theorem 1.2.

\section{Proof of Theorem 1.2}

First, we give the following well-known Gagliardo-Nirenberg inequality that will be used frequently.

Lemma 2.1 For $p \in[2,6], q \in(1, \infty)$ and $r \in(3, \infty)$, there exists some generic constant $C>0$, which may depend on $p, q$ and $r$, such that for any $f \in H^{1}$ and $g \in L^{q} \cap D^{1, r}$, we have

$$
\begin{aligned}
& \|f\|_{L^{p}} \leq C\|f\|_{L^{2}}^{(6-p) / 2 p}\|\nabla f\|_{L^{2}}^{(3 p-6) / 2 p}, \\
& \|g\|_{L^{\infty}} \leq C\|g\|_{L^{q}}^{q(r-3) /(3 r+q(r-3))}\|\nabla g\|_{L^{r}}^{3 r /(3 r+q(r-3))} .
\end{aligned}
$$

The following BKM's type inequality which will be used to estimate $\|\nabla u\|_{L^{\infty}}$ and $\|\nabla \rho\|_{L^{q}}$ with $q \in(3,6]$ can be found in [12].

Lemma 2.2 For $3<q<\infty$, there is a constant $C=C(q)$, depending only on $q$, such that the following estimate holds for all $\nabla u \in L^{2} \cap D^{1, q}$ :

$$
\|\nabla u\|_{L^{\infty}} \leq C\left(\|\operatorname{div} u\|_{L^{\infty}}+\|\nabla \times u\|_{L^{\infty}}\right) \log \left(e+\left\|\nabla^{2} u\right\|_{L^{q}}\right)+C\|\nabla u\|_{L^{2}}+C .
$$

The proof of Theorem 1.2 is based on the contradiction arguments. Let $(\rho, u, w, H)$ be a strong solution of the problem (1.1), (1.2) and (1.4) as described in Theorem 1.1. Suppose that (1.9) is false, that is,

$$
\lim _{T \rightarrow T^{*}}\left(\|\rho\|_{L^{\infty}\left(0, T ; L^{\infty}\right)}+\|u\|_{L^{s}\left(0, T ; L^{r}\right)}\right) \leq M_{0}<\infty
$$

where $r, s$ satisfy (1.3) and $M_{0}$ is a constant.

One can easily deduce from the following energy estimate (1.1), (1.2) and (1.4). 
Lemma 2.3 It holds that

$$
\begin{gathered}
\sup _{0 \leq t \leq T}\left(\|\rho\|_{L^{1} \cap L^{\gamma}}+\|\sqrt{\rho} u\|_{L^{2}}^{2}+\|\sqrt{\rho} w\|_{L^{2}}^{2}+\|H\|_{L^{2}}^{2}\right) \\
+\int_{0}^{T}\left(\|\nabla u\|_{L^{2}}^{2}+\|w\|_{H^{1}}^{2}+\|\nabla H\|_{L^{2}}^{2}\right) \mathrm{d} t \leq C .
\end{gathered}
$$

Here and hereafter, $C$ denotes a generic positive constant which may depend on $\mu, \mu^{\prime}, \lambda, \lambda^{\prime}$, $\xi, \sigma, A, \gamma, \rho_{0}, u_{0}, w_{0}, H_{0}, g_{1}, g_{2}, T$ and $M_{0}$.

We denote the material derivative of $f$ by $\dot{f}=f_{t}+u \cdot \nabla f$ and set

$$
\begin{aligned}
& G_{1}:=(2 \mu+\lambda) \operatorname{div} u-P(\rho)-\frac{1}{2}|H|^{2}, \quad G_{2}:=\left(2 \mu^{\prime}+\lambda^{\prime}\right) \operatorname{div} w \\
& V_{1}:=\nabla \times u, \quad V_{2}:=\nabla \times w .
\end{aligned}
$$

Since $(\nabla \times H) \times H=H \cdot \nabla H-\frac{1}{2} \nabla|H|^{2}$ due to $(1.1)_{5}$, we have from $(1.1)_{2}$ and $(1.1)_{3}$ that

$$
\left\{\begin{array}{l}
\triangle G_{1}=\operatorname{div}(\rho \dot{u}-H \cdot \nabla H), \\
\triangle G_{2}-\frac{4 \xi}{2 \mu^{\prime}+\lambda^{\prime}} G_{2}=\operatorname{div}(\rho \dot{w}), \\
(\mu+\xi) \triangle V_{1}=\nabla \times(\rho \dot{u}-H \cdot \nabla H)-2 \xi \nabla \times V_{2}, \\
\mu^{\prime} \triangle V_{2}-4 \xi V_{2}=\nabla \times(\rho \dot{w})-2 \xi \nabla \times V_{1} .
\end{array}\right.
$$

Thus, from the standard $L^{p}$-estimate of an elliptic system, we have the following lemma.

Lemma 2.4 Under the condition (2.4), it holds that

$$
\begin{aligned}
& \left\|\nabla G_{1}\right\|_{L^{2}}+\left\|\nabla V_{1}\right\|_{L^{2}}+\left\|G_{2}\right\|_{H^{1}}+\left\|V_{2}\right\|_{H^{1}} \\
& \leq C\left(\|\sqrt{\rho} \dot{u}\|_{L^{2}}+\|\sqrt{\rho} \dot{w}\|_{L^{2}}+\|\nabla u\|_{L^{2}}+\|\nabla w\|_{L^{2}}+\|H \nabla H\|_{L^{2}}\right) \\
& \leq C\left(\left\|\sqrt{\rho} u_{t}\right\|_{L^{2}}+\left\|\sqrt{\rho} w_{t}\right\|_{L^{2}}+\|u \nabla u\|_{L^{2}}+\|w \nabla w\|_{L^{2}}+\|\nabla u\|_{L^{2}}+\|\nabla w\|_{L^{2}}\right. \\
& \left.\quad+\|H \nabla H\|_{L^{2}}\right), \\
& \left\|\nabla G_{1}\right\|_{L^{6}}+\left\|\nabla G_{2}\right\|_{L^{6}}+\left\|\nabla V_{1}\right\|_{L^{6}}+\left\|\nabla V_{2}\right\|_{L^{6}} \\
& \leq C\left(\|\nabla \dot{u}\|_{L^{2}}+\|\nabla \dot{w}\|_{L^{2}}+\|\sqrt{\rho} \dot{u}\|_{L^{2}}+\|\sqrt{\rho} \dot{w}\|_{L^{2}}+\|\nabla u\|_{L^{2}}+\|\nabla w\|_{L^{2}}+\|H \nabla H\|_{L^{2}}\right. \\
& \left.\quad+\|H \nabla H\|_{L^{6}}\right) .
\end{aligned}
$$

Proof In view of standard $L^{2}$-estimates of elliptic system (2.7), one immediately obtains (2.8). By (2.1) and (2.4), we get that

$$
\begin{aligned}
& \left\|\nabla G_{1}\right\|_{L^{6}}+\left\|\nabla G_{2}\right\|_{L^{6}}+\left\|\nabla V_{1}\right\|_{L^{6}}+\left\|\nabla V_{2}\right\|_{L^{6}} \\
& \quad \leq C\left(\|\rho \dot{u}\|_{L^{6}}+\|\rho \dot{w}\|_{L^{6}}+\left\|G_{2}\right\|_{L^{2}}+\left\|V_{1}\right\|_{L^{6}}+\left\|V_{2}\right\|_{L^{2}}+\left\|V_{2}\right\|_{L^{6}}+\|H \nabla H\|_{L^{6}}\right) \\
& \quad \leq C\left(\|\nabla \dot{u}\|_{L^{2}}+\|\nabla \dot{w}\|_{L^{2}}+\left\|G_{2}\right\|_{L^{2}}+\left\|\nabla V_{1}\right\|_{L^{2}}+\left\|V_{2}\right\|_{H^{1}}+\|H \nabla H\|_{L^{6}}\right),
\end{aligned}
$$

which, combined with (2.8), yields (2.9) immediately. 
The next lemma is concerned with the higher integrability of $H$ under the assumption (2.4).

Lemma 2.5 Under the condition (2.4), it holds for any $0 \leq T \leq T^{*}$ that

$$
\|H\|_{L^{\infty}\left(0, T ; L^{q}\right)} \leq C(q) \quad \text { for any } q \in[2, \infty)
$$

where $C(q)$ is a positive constant depending on $q$.

The proof is similar to Lemma 3.3 in [12] and is omitted here.

With the help of (2.4) and Lemmas 2.3-2.5, we can prove the following key lemma.

Lemma 2.6 Under the condition (2.4), it holds that for any $0 \leq T<T^{*}$,

$$
\begin{aligned}
& \sup _{0 \leq t \leq T}\left(\|\nabla u\|_{L^{2}}^{2}+\|w\|_{H^{1}}^{2}+\|\nabla H\|_{L^{2}}^{2}\right) \\
& \quad+\int_{0}^{T}\left(\left\|\sqrt{\rho} u_{t}\right\|_{L^{2}}^{2}+\left\|\sqrt{\rho} w_{t}\right\|_{L^{2}}^{2}+\left\|H_{t}\right\|_{L^{2}}^{2}+\|\nabla H\|_{H^{1}}^{2}\right) \mathrm{d} t \leq C .
\end{aligned}
$$

Proof Multiplying $(1.1)_{2},(1.1)_{3}$ and $(1.1)_{4}$ by $u_{t}, w_{t}$ and $H_{t}$, respectively, and integrating the resulting equations by parts, one obtains after summing up that

$$
\begin{aligned}
& \frac{1}{2} \frac{\mathrm{d}}{\mathrm{d} t} \int\left(\mu|\nabla u|^{2}+(\mu+\lambda)(\operatorname{div} u)^{2}+\mu^{\prime}|\nabla w|^{2}+\left(\mu^{\prime}+\lambda^{\prime}\right)(\operatorname{div} w)^{2}+\sigma|\nabla H|^{2}\right) \mathrm{d} x \\
& \quad+\frac{1}{2} \frac{\mathrm{d}}{\mathrm{d} t} \int \xi\left(|\nabla \times u|^{2}+4|w|^{2}\right) \mathrm{d} x+\int\left(\rho\left|u_{t}\right|^{2}+\rho\left|w_{t}\right|^{2}+\left|H_{t}\right|^{2}\right) \mathrm{d} x \\
& =\int P \operatorname{div} u_{t} \mathrm{~d} x+2 \xi \int\left(\nabla \times w \cdot u_{t}+\nabla \times u \cdot w_{t}\right) \mathrm{d} x \\
& \quad-\int\left(\rho u \cdot \nabla u \cdot u_{t}+\rho u \cdot \nabla w \cdot w_{t}\right) \mathrm{d} x+\int\left(H \cdot \nabla H-\frac{1}{2} \nabla|H|^{2}\right) \cdot u_{t} \mathrm{~d} x \\
& \quad+\int H \cdot \nabla u \cdot H_{t} \mathrm{~d} x-\int u \cdot \nabla H \cdot H_{t} \mathrm{~d} x-\int H \cdot H_{t} \operatorname{div} u \mathrm{~d} x:=\sum_{i=1}^{7} I_{i} .
\end{aligned}
$$

To estimate the first term on the right-hand side of (2.12), we observe that $P$ satisfies

$$
P_{t}+\operatorname{div}(P u)+(\gamma-1) P \operatorname{div} u=0 .
$$

Hence, using (2.4), (2.5) and (2.10) yields that

$$
\begin{aligned}
I_{1}= & \frac{\mathrm{d}}{\mathrm{d} t} \int P \operatorname{div} u \mathrm{~d} x-\int P u \cdot \nabla \operatorname{div} u \mathrm{~d} x+(\gamma-1) \int P(\operatorname{div} u)^{2} \mathrm{~d} x \\
= & \frac{\mathrm{d}}{\mathrm{d} t} \int P \operatorname{div} u \mathrm{~d} x-\frac{1}{2 \mu+\lambda} \int P u \cdot \nabla G_{1} \mathrm{~d} x+\frac{1}{2(2 \mu+\lambda)} \int P^{2} \operatorname{div} u \mathrm{~d} x \\
& -\frac{1}{2(2 \mu+\lambda)} \int P u \cdot \nabla|H|^{2} \mathrm{~d} x+(\gamma-1) \int P(\operatorname{div} u)^{2} \mathrm{~d} x \\
\leq & \frac{\mathrm{d}}{\mathrm{d} t} \int P \operatorname{div} u \mathrm{~d} x+C\left(\|P\|_{L^{3}}\|u\|_{L^{6}}\left\|\nabla G_{1}\right\|_{L^{2}}+\|P\|_{L^{\infty}}\|P\|_{L^{2}}\|\nabla u\|_{L^{2}}\right.
\end{aligned}
$$




$$
\begin{aligned}
& \left.+\|P\|_{L^{\infty}}\|u\|_{L^{6}}\|H\|_{L^{3}}\|\nabla H\|_{L^{2}}+\|P\|_{L^{\infty}}\|\nabla u\|_{L^{2}}^{2}\right) \\
\leq & \frac{\mathrm{d}}{\mathrm{d} t} \int P \operatorname{div} u \mathrm{~d} x+\varepsilon\left\|\nabla G_{1}\right\|_{L^{2}}^{2}+C(\varepsilon)\left(\|\nabla u\|_{L^{2}}^{2}+\|\nabla H\|_{L^{2}}^{2}+1\right),
\end{aligned}
$$

where we have used Young's inequality and (2.1).

For the second term, we have, after integration by parts, that

$$
I_{2}=2 \xi \int\left(w \cdot\left(\nabla \times u_{t}\right)+(\nabla \times u) \cdot w_{t}\right) \mathrm{d} x=2 \xi \frac{\mathrm{d}}{\mathrm{d} t} \int w \cdot(\nabla \times u) \mathrm{d} x
$$

and by the Cauchy-Schwarz inequality, we have

$$
I_{3} \leq \frac{1}{4}\left(\left\|\sqrt{\rho} u_{t}\right\|_{L^{2}}^{2}+\left\|\sqrt{\rho} w_{t}\right\|_{L^{2}}^{2}\right)+C\left(\|u \nabla u\|_{L^{2}}^{2}+\|u \nabla w\|_{L^{2}}^{2}\right) .
$$

Similarly, integrating by parts and using the fact $\operatorname{div} H=0$, one has

$$
\begin{aligned}
I_{4}= & -\int\left(H \cdot \nabla u_{t} \cdot H-\frac{1}{2}|H|^{2} \operatorname{div} u_{t}\right) \mathrm{d} x \\
= & -\frac{\mathrm{d}}{\mathrm{d} t} \int\left(H \cdot \nabla u \cdot H-\frac{1}{2}|H|^{2} \operatorname{div} u\right) \mathrm{d} x \\
& +\int\left(H_{t} \cdot \nabla u \cdot H+H \cdot \nabla u \cdot H_{t}-H \cdot H_{t} \operatorname{div} u\right) \mathrm{d} x \\
\leq & -\frac{\mathrm{d}}{\mathrm{d} t} \int\left(H \cdot \nabla u \cdot H-\frac{1}{2}|H|^{2} \operatorname{div} u\right) \mathrm{d} x+\frac{1}{4}\left\|H_{t}\right\|_{L^{2}}^{2}+C\|H \nabla u\|_{L^{2}}^{2} .
\end{aligned}
$$

For the last three terms on the right-hand side of (2.12), one has from (2.4) that

$$
\left|\sum_{i=5}^{7} I_{i}\right| \leq \frac{1}{4}\left\|H_{t}\right\|_{L^{2}}^{2}+C\left(\|u \nabla H\|_{L^{2}}^{2}+\|H \nabla u\|_{L^{2}}^{2}\right) .
$$

Thus, putting (2.13)-(2.17) into (2.12) and choosing $\varepsilon>0$ suitably small, we infer from (2.8) that

$$
\begin{aligned}
& \frac{\mathrm{d}}{\mathrm{d} t} \int\left(\mu|\nabla u|^{2}+(\mu+\lambda)(\operatorname{div} u)^{2}+\mu^{\prime}|\nabla w|^{2}+\left(\mu^{\prime}+\lambda^{\prime}\right)(\operatorname{div} w)^{2}+\sigma|\nabla H|^{2}\right) \mathrm{d} x \\
& \quad+\frac{\mathrm{d}}{\mathrm{d} t} \int \xi|\nabla \times u-2 w|^{2} \mathrm{~d} x+\int\left(\rho\left|u_{t}\right|^{2}+\rho\left|w_{t}\right|^{2}+\left|H_{t}\right|^{2}\right) \mathrm{d} x \\
& \leq \frac{\mathrm{d}}{\mathrm{d} t} \int\left(2 P \operatorname{div} u+|H|^{2} \operatorname{div} u-2 H \cdot \nabla u \cdot H\right) \mathrm{d} x+C\left(\|\nabla u\|_{L^{2}}^{2}+\|\nabla w\|_{L^{2}}^{2}+\|\nabla H\|_{L^{2}}^{2}\right) \\
& \quad+C\left(\|u \nabla u\|_{L^{2}}^{2}+\|u \nabla w\|_{L^{2}}^{2}+\|u \nabla H\|_{L^{2}}^{2}+\|H \nabla u\|_{L^{2}}^{2}+\|H \nabla H\|_{L^{2}}^{2}\right) .
\end{aligned}
$$

For any $r, s$ satisfying (1.3), we have by the Hölder and Sobolev inequalities that

$$
\begin{aligned}
\|f g\|_{L^{2}} & \leq C\|f\|_{L^{r}}\|g\|_{L^{\frac{2 r}{r-2}}} \leq C\|f\|_{L^{r}}\|g\|_{L^{2}}^{\frac{r-3}{r}}\|g\|_{L^{6}}^{\frac{3}{r}} \\
& \leq \delta\|g\|_{L^{6}}+C(\delta)\left(\|f\|_{L^{r}}^{\frac{5}{2}}+1\right)\|g\|_{L^{2}}, \quad r>3,
\end{aligned}
$$

for some $\delta \in(0,1)$. 
Taking $f=u, H, g=\nabla u, \nabla w, \nabla H$ into (2.19) and using (2.10), we obtain

$$
\begin{gathered}
\|u \nabla u\|_{L^{2}}^{2}+\|u \nabla w\|_{L^{2}}^{2}+\|u \nabla H\|_{L^{2}}^{2}+\|H \nabla u\|_{L^{2}}^{2}+\|H \nabla H\|_{L^{2}}^{2} \\
\leq \delta\left(\|\nabla u\|_{L^{6}}^{2}+\|\nabla w\|_{L^{6}}^{2}+\|\nabla H\|_{L^{6}}^{2}\right) \\
\quad+C(\delta)\left(\|u\|_{L^{r}}^{s}+1\right)\left(\|\nabla u\|_{L^{2}}^{2}+\|\nabla w\|_{L^{2}}^{2}+\|\nabla H\|_{L^{2}}^{2}\right) .
\end{gathered}
$$

By the standard $L^{p}$-estimate, one can deduce from (2.1), (2.4), (2.8) and (2.10) that

$$
\begin{aligned}
\| & \nabla u\left\|_{L^{6}}+\right\| \nabla w \|_{L^{6}} \\
& \leq C\left(\|\operatorname{div} u\|_{L^{6}}+\|\nabla \times u\|_{L^{6}}+\|\operatorname{div} w\|_{L^{6}}+\|\nabla \times w\|_{L^{6}}\right) \\
& \leq C\left(\left\|G_{1}\right\|_{L^{6}}+\left\|G_{2}\right\|_{L^{6}}+\left\|V_{1}\right\|_{L^{6}}+\left\|V_{2}\right\|_{L^{6}}+\|P\|_{L^{6}}+\left\||H|^{2}\right\|_{L^{6}}\right) \\
& \leq C\left(1+\left\|\nabla G_{1}\right\|_{L^{2}}+\left\|\nabla G_{2}\right\|_{L^{2}}+\left\|\nabla V_{1}\right\|_{L^{2}}+\left\|\nabla V_{2}\right\|_{L^{2}}\right) \\
& \leq C\left(1+\left\|\sqrt{\rho} u_{t}\right\|_{L^{2}}+\left\|\sqrt{\rho} w_{t}\right\|_{L^{2}}\right)+C\left(\|u \nabla u\|_{L^{2}}+\|u \nabla u\|_{L^{2}}+\|H \nabla H\|_{L^{2}}\right) .
\end{aligned}
$$

Furthermore, it follows from $(1.1)_{4}$ and Sobolev's embedding inequality that

$$
\|\nabla H\|_{L^{6}} \leq C\|\nabla H\|_{H^{1}} \leq C\left(\left\|H_{t}\right\|_{L^{2}}+\|u \nabla H\|_{L^{2}}+\|H \nabla u\|_{L^{2}}+\|\nabla H\|_{L^{2}}\right),
$$

putting (2.21) and (2.22) into (2.20), such that

$$
\begin{gathered}
\|u \nabla u\|_{L^{2}}^{2}+\|u \nabla w\|_{L^{2}}^{2}+\|u \nabla H\|_{L^{2}}^{2}+\|H \nabla u\|_{L^{2}}^{2}+\|H \nabla H\|_{L^{2}}^{2} \\
\leq C \delta\left(\left\|\sqrt{\rho} u_{t}\right\|_{L^{2}}^{2}+\left\|\sqrt{\rho} w_{t}\right\|_{L^{2}}^{2}+\left\|H_{t}\right\|_{L^{2}}^{2}\right) \\
\quad+C(\delta)\left(\|u\|_{L^{r}}^{s}+1\right)\left(\|\nabla u\|_{L^{2}}^{2}+\|\nabla w\|_{L^{2}}^{2}+\|\nabla H\|_{L^{2}}^{2}\right),
\end{gathered}
$$

which, together (2.21) and (2.18), choosing $\delta>0$ suitably small, gives

$$
\begin{aligned}
& \frac{\mathrm{d}}{\mathrm{d} t} \int\left(\mu|\nabla u|^{2}+(\mu+\lambda)(\operatorname{div} u)^{2}+\mu^{\prime}|\nabla w|^{2}+\left(\mu^{\prime}+\lambda^{\prime}\right)(\operatorname{div} w)^{2}+\sigma|\nabla H|^{2}\right) \mathrm{d} x \\
& \quad+\frac{\mathrm{d}}{\mathrm{d} t} \int \xi|\nabla \times u-2 w|^{2} \mathrm{~d} x+\int\left(\rho\left|u_{t}\right|^{2}+\rho\left|w_{t}\right|^{2}+\left|H_{t}\right|^{2}\right) \mathrm{d} x \\
& \leq \frac{\mathrm{d}}{\mathrm{d} t} \int\left(2 P \operatorname{div} u+|H|^{2} \operatorname{div} u-2 H \cdot \nabla u \cdot H\right) \mathrm{d} x \\
& \quad+C\left(\|u\|_{L^{r}}^{s}+1\right)\left(\|\nabla u\|_{L^{2}}^{2}+\|\nabla w\|_{L^{2}}^{2}+\|\nabla H\|_{L^{2}}^{2}\right) .
\end{aligned}
$$

It is easily seen that

$$
\left|\int\left(2 P \operatorname{div} u+|H|^{2} \operatorname{div} u-2 H \cdot \nabla u \cdot H\right) \mathrm{d} x\right| \leq \frac{\mu}{4}\|\nabla u\|_{L^{2}}^{2}+C .
$$

Taking this into account, we conclude from (2.4), (2.24) and Gronwall's inequality that part of (2.11) holds for any $0 \leq T<T^{*}$. Note that the estimate of $\|\nabla H\|_{L^{2}\left(0, T ; H^{1}\right)}$ is a consequence of (2.4), (2.22) and (2.23). The proof of this lemma is completed.

Next we prove the boundedness of $\|\sqrt{\rho} \dot{u}\|_{L^{2}},\|\sqrt{\rho} \dot{w}\|_{L^{2}},\left\|H_{t}\right\|_{L^{2}}$ and $\|\nabla H\|_{H^{1}}$ by the compatibility conditions (1.6) and (1.7). 
Lemma 2.7 Under the condition (2.4), it holds that for any $0 \leq T<T^{*}$,

$$
\begin{gathered}
\sup _{0 \leq t \leq T}\left(\|\sqrt{\rho} \dot{u}\|_{L^{2}}^{2}+\|\sqrt{\rho} \dot{w}\|_{L^{2}}^{2}+\left\|H_{t}\right\|_{L^{2}}^{2}+\|\nabla H\|_{H^{1}}^{2}\right) \\
\quad+\int_{0}^{T}\left(\|\nabla \dot{u}\|_{L^{2}}^{2}+\|\nabla \dot{w}\|_{L^{2}}^{2}+\left\|\nabla H_{t}\right\|_{L^{2}}^{2}\right) \mathrm{d} t \leq C .
\end{gathered}
$$

Proof Applying the operator $\dot{u}^{j}\left[\partial_{t}+\operatorname{div}(u \cdot)\right]$ and $\dot{u}^{j}\left[\partial_{t}+\operatorname{div}(u \cdot)\right]$ to both sides of $(1.1)_{2}$ and $(1.1)_{3}$, respectively, and using $(1.1)_{1}$, one can obtain, after a straightforward calculation, that

$$
\begin{aligned}
\frac{1}{2} \frac{\mathrm{d}}{\mathrm{d} t} & \int\left(\rho|\dot{u}|^{2}+\rho|\dot{w}|^{2}\right) \mathrm{d} x \\
= & (\mu+\xi) \int \dot{u}^{j}\left[\Delta u_{t}^{j}+\operatorname{div}\left(u \Delta u^{j}\right)\right] \mathrm{d} x+(\mu+\lambda-\xi) \int \dot{u}^{j}\left[\partial_{t} \partial_{j} \operatorname{div} u+\operatorname{div}\left(u \partial_{j} \operatorname{div} u\right)\right] \mathrm{d} x \\
& +\mu^{\prime} \int \dot{w}^{j}\left[\Delta w_{t}^{j}+\operatorname{div}\left(u \Delta w^{j}\right)\right] \mathrm{d} x+\left(\mu^{\prime}+\lambda^{\prime}\right) \int \dot{w}^{j}\left[\partial_{t} \partial_{j} \operatorname{div} w+\operatorname{div}\left(u \partial_{j} \operatorname{div} w\right)\right] \mathrm{d} x \\
& \quad-\int \dot{u}^{j}\left[\partial_{j} P_{t}+\operatorname{div}\left(u \partial_{j} P\right)\right] \mathrm{d} x+2 \xi \int \dot{u} \cdot\left[\nabla \times w_{t}+\partial_{i}\left(u^{i} \nabla \times w\right)\right] \mathrm{d} x \\
& +2 \xi \int \dot{w} \cdot\left[\nabla \times u_{t}+\partial_{i}\left(u^{i} \nabla \times u\right)\right] \mathrm{d} x-4 \xi \int \dot{w}^{j}\left[w_{t}^{j}+\operatorname{div}\left(u w^{j}\right)\right] \mathrm{d} x \\
& \quad-\frac{1}{2} \int \dot{u}^{j}\left[\partial_{t} \partial_{j}|H|^{2}+\operatorname{div}\left(u \partial_{j}|H|^{2}\right)\right] \mathrm{d} x+\int \dot{u}^{j}\left[\partial_{t}\left(H \cdot \nabla H^{j}\right)+\operatorname{div}\left(u\left(H \cdot \nabla H^{j}\right)\right)\right] \mathrm{d} x \\
:= & \sum_{i=1}^{10} J_{i} .
\end{aligned}
$$

We get after integration by parts that

$$
\begin{aligned}
J_{1} & =-(\mu+\xi) \int\left(\left|\nabla \dot{u}^{j}\right|^{2}-\partial_{i} \dot{u}^{j} \partial_{i} u^{k} \partial_{k} u^{j}-\partial_{i} \dot{u}^{j} u^{k} \partial_{k} \partial_{i} u^{j}+u \cdot \nabla \dot{u}^{j} \Delta u^{j}\right) \mathrm{d} x \\
& =-(\mu+\xi) \int\left(\left|\nabla \dot{u}^{j}\right|^{2}-\partial_{i} \dot{u}^{j} \partial_{i} u^{k} \partial_{k} u^{j}+\partial_{i} \dot{u}^{j} \partial_{k} u^{k} \partial_{i} u^{j}-\partial_{i} u^{k} \partial_{k} \dot{u}^{j} \partial_{i} u^{j}\right) \mathrm{d} x \\
& \leq-(\mu+\xi)\|\nabla \dot{u}\|_{L^{2}}^{2}+C\|\nabla \dot{u}\|_{L^{2}}\|\nabla u\|_{L^{4}}^{2} .
\end{aligned}
$$

Similarly, we also have

$$
\begin{aligned}
\sum_{j=2}^{4} J_{j} \leq & -(\mu+\lambda-\xi)\|\operatorname{div} \dot{u}\|_{L^{2}}^{2}-\mu^{\prime}\|\nabla \dot{w}\|_{L^{2}}^{2}-\left(\mu^{\prime}+\lambda^{\prime}\right)\|\operatorname{div} \dot{w}\|_{L^{2}}^{2} \\
& +C\|\nabla \dot{u}\|_{L^{2}}\|\nabla u\|_{L^{4}}^{2}+C\|\nabla \dot{w}\|_{L^{2}}\|\nabla u\|_{L^{4}}\|\nabla w\|_{L^{4}} .
\end{aligned}
$$

After integration by parts, using $(1.1)_{1}$ and (2.11), we obtain

$$
\begin{aligned}
J_{5} & =-\int\left(\rho P^{\prime}(\rho) \operatorname{div} u \operatorname{div} \dot{u}-P(\rho) \partial_{k}\left(u^{k} \partial_{j} \dot{u}^{j}\right)-P(\rho) \partial_{j}\left(u^{k} \partial_{k} \dot{u}^{j}\right)\right) \mathrm{d} x \\
& \leq C\|\nabla u\|_{L^{2}}\|\nabla \dot{u}\|_{L^{2}} \leq C\|\nabla \dot{u}\|_{L^{2}} .
\end{aligned}
$$


Using the definition of the material derivation and integrating by parts, we deduce from (2.1), (2.5) and (2.11) that

$$
\begin{aligned}
J_{6} & =2 \xi \int\left[w_{t} \cdot(\nabla \times \dot{u})-u \cdot \nabla \dot{u} \cdot(\nabla \times w)\right] \mathrm{d} x \\
& =2 \xi \int \dot{w} \cdot(\nabla \times \dot{u}) \mathrm{d} x-2 \xi \int[u \cdot \nabla w \cdot(\nabla \times \dot{u})+u \cdot \nabla \dot{u} \cdot(\nabla \times w)] \mathrm{d} x \\
& \leq 2 \xi \int \dot{w} \cdot(\nabla \times \dot{u}) \mathrm{d} x+C\|u\|_{L^{6}}\|\nabla w\|_{L^{3}}\|\nabla \dot{u}\|_{L^{2}} \\
& \leq 2 \xi \int \dot{w} \cdot(\nabla \times \dot{u}) \mathrm{d} x+C\|\nabla w\|_{L^{3}}\|\nabla \dot{u}\|_{L^{2}},
\end{aligned}
$$

and, similarly,

$$
\begin{aligned}
J_{7} & =2 \xi \int \dot{w} \cdot(\nabla \times \dot{u}) \mathrm{d} x-2 \xi \int[u \cdot \nabla u \cdot(\nabla \times \dot{w})+u \cdot \nabla \dot{w} \cdot(\nabla \times u)] \mathrm{d} x \\
& \leq 2 \xi \int \dot{w} \cdot(\nabla \times \dot{u}) \mathrm{d} x+C\|\nabla u\|_{L^{3}}\|\nabla \dot{w}\|_{L^{2}},
\end{aligned}
$$

and

$$
\begin{aligned}
J_{8} & =-4 \xi \int|\dot{w}|^{2} \mathrm{~d} x+4 \xi \int[u \cdot \nabla w \cdot \dot{w}+u \cdot \nabla \dot{w} \cdot w] \mathrm{d} x \\
& =-4 \xi \int|\dot{w}|^{2} \mathrm{~d} x-4 \xi \int w \cdot \dot{w} \operatorname{div} u \mathrm{~d} x \\
& \leq-4 \xi \int|\dot{w}|^{2} \mathrm{~d} x+C\|\nabla u\|_{L^{2}}\|w\|_{L^{3}}\|\dot{w}\|_{L^{6}} \\
& \leq-4 \xi \int|\dot{w}|^{2} \mathrm{~d} x+C\|\nabla \dot{w}\|_{L^{2}} .
\end{aligned}
$$

The ninth term on the right-hand side of (2.26) can be estimated as follows, integrating by parts, using (2.1), (2.5), (2.10), (2.11) and Hölder's inequality:

$$
\begin{aligned}
J_{9} & =\int\left(\partial_{j} \dot{u}^{j} H \cdot H_{t}+\partial_{k} \dot{u}^{j} u^{k} \partial_{j} H \cdot H\right) \mathrm{d} x \\
& \leq C\|\nabla \dot{u}\|_{L^{2}}\left(\|H\|_{L^{6}}\left\|H_{t}\right\|_{L^{3}}+\|u\|_{L^{6}}\|H\|_{L^{6}}\|\nabla H\|_{L^{6}}\right) \\
& \leq C\|\nabla \dot{u}\|_{L^{2}}\left(\left\|H_{t}\right\|_{L^{2}}^{\frac{1}{2}}\left\|H_{t}\right\|_{L^{6}}^{\frac{1}{2}}+\|\nabla u\|_{L^{2}}\|\nabla H\|_{H^{1}}\right) \\
& \leq \delta\|\nabla \dot{u}\|_{L^{2}}^{2}+C(\delta)\left(\left\|H_{t}\right\|_{L^{2}}^{2}+\left\|\nabla H_{t}\right\|_{L^{2}}^{2}+\|\nabla H\|_{H^{1}}^{2}\right) .
\end{aligned}
$$

In a similar manner, one also has

$$
\begin{aligned}
J_{10} & =\int \dot{u}^{j}\left(H_{t} \cdot \nabla H^{j}+H \cdot \nabla H_{t}^{j}+\operatorname{div}\left(u\left(H \cdot \nabla H^{j}\right)\right)\right) \mathrm{d} x \\
& =-\int\left(\left(H_{t} \cdot \nabla \dot{u}^{j}\right) H^{j}+\left(H \cdot \nabla \dot{u}^{j}\right) H_{t}^{j}+u^{k} \partial_{k} \dot{u}^{j}\left(H \cdot \nabla H^{j}\right)\right) \mathrm{d} x \\
& \leq \delta\|\nabla \dot{u}\|_{L^{2}}^{2}+C(\delta)\left(\left\|H_{t}\right\|_{L^{2}}^{2}+\left\|\nabla H_{t}\right\|_{L^{2}}^{2}+\|\nabla H\|_{H^{1}}^{2}\right) .
\end{aligned}
$$


Putting (2.27)-(2.34) into (2.26), using the Cauchy-Schwarz inequality and choosing $\delta>0$ suitably small, we get

$$
\begin{aligned}
& \frac{\mathrm{d}}{\mathrm{d} t}\left(\|\sqrt{\rho} \dot{\mu}\|_{L^{2}}^{2}+\|\sqrt{\rho} \dot{w}\|_{L^{2}}^{2}\right)+\left(\|\nabla \dot{u}\|_{L^{2}}^{2}+\|\nabla \dot{w}\|_{L^{2}}^{2}+\|2 \dot{w}-\nabla \times \dot{u}\|_{L^{2}}^{2}\right) \\
& \quad \leq C\left(1+\|\nabla u\|_{L^{6}}^{3}+\|\nabla w\|_{L^{6}}^{3}+\left\|H_{t}\right\|_{L^{2}}^{2}+\left\|\nabla H_{t}\right\|_{L^{2}}^{2}+\|\nabla H\|_{H^{1}}^{2}\right) .
\end{aligned}
$$

To estimate $\left\|H_{t}\right\|_{L^{2}}$, one can differentiate (1.1) $)_{4}$ with respect to $t$, multiply the resulting equations by $H_{t}$ in $L^{2}$, and integrate by parts over $\mathbb{R}^{3}$ to get

$$
\begin{aligned}
\frac{1}{2} & \frac{\mathrm{d}}{\mathrm{d} t} \int\left|H_{t}\right|^{2} \mathrm{~d} x+\sigma \int\left|\nabla H_{t}\right|^{2} \mathrm{~d} x \\
= & \int\left(H \cdot \nabla u_{t}-u_{t} \cdot \nabla H-H \operatorname{div} u_{t}\right) \cdot H_{t} \mathrm{~d} x \\
& \quad+\int\left(H_{t} \cdot \nabla u-u \cdot \nabla H_{t}-H_{t} \operatorname{div} u\right) \cdot H_{t} \mathrm{~d} x:=K_{1}+K_{2} .
\end{aligned}
$$

Integrating by parts and using $(1.1)_{5},(2.1),(2.10)$ and (2.11), then we deduce

$$
\begin{aligned}
K_{1}= & \int(H \cdot \nabla \dot{u}-\dot{u} \nabla H-H \operatorname{div} \dot{u}) \cdot H_{t} \mathrm{~d} x+\int\left(H^{i} \partial_{i} H_{t}^{j}-H^{k} \partial_{j} H_{t}^{k}\right)\left(u \cdot \nabla u^{j}\right) \mathrm{d} x \\
\leq & C\|H\|_{L^{6}}\left\|H_{t}\right\|_{L^{3}}\|\nabla \dot{u}\|_{L^{2}}+C\|\dot{u}\|_{L^{6}}\|\nabla H\|_{L^{2}}\left\|H_{t}\right\|_{L^{3}} \\
& +C\|H\|_{L^{12}}\left\|\nabla H_{t}\right\|_{L^{2}}\|\nabla u\|_{L^{4}}\|u\|_{L^{6}} \\
\leq & C\left(\left\|H_{t}\right\|_{L^{2}}^{\frac{1}{2}}\left\|\nabla H_{t}\right\|_{L^{2}}^{\frac{1}{2}}\|\nabla \dot{u}\|_{L^{2}}+\left\|\nabla H_{t}\right\|_{L^{2}}\|\nabla u\|_{L^{4}}\right) \\
\leq & \varepsilon_{1}\left\|\nabla H_{t}\right\|_{L^{2}}^{2}+\varepsilon_{2}\|\nabla \dot{u}\|_{L^{2}}^{2}+C\left(\varepsilon_{1}, \varepsilon_{2}\right)\left(\left\|H_{t}\right\|_{L^{2}}^{2}+\|\nabla u\|_{L^{4}}^{2}\right)
\end{aligned}
$$

for some positive constants $\varepsilon_{1}, \varepsilon_{2} \in(0,1)$. For the second term on the right-hand side of (2.36), integrating by parts and using (2.1) give

$$
\begin{aligned}
K_{2} & =\int\left(H_{t} \cdot \nabla u-\frac{1}{2} H_{t} \operatorname{div} u\right) \cdot H_{t} \mathrm{~d} x \leq C\|\nabla u\|_{L^{2}}\left\|H_{t}\right\|_{L^{2}} \\
& \leq C\left\|H_{t}\right\|_{L^{2}}^{\frac{1}{2}}\left\|\nabla H_{t}\right\|_{L^{2}}^{\frac{3}{2}} \leq \varepsilon_{1}\left\|\nabla H_{t}\right\|_{L^{2}}^{2}+C\left(\varepsilon_{1}\right)\left\|H_{t}\right\|_{L^{2}}^{2} .
\end{aligned}
$$

Putting the estimates of $K_{1}, K_{2}$ into (2.36) and choosing $\varepsilon_{1}>0$ small enough, one has

$$
\frac{1}{2} \frac{\mathrm{d}}{\mathrm{d} t} \int\left|H_{t}\right|^{2} \mathrm{~d} x+\frac{\sigma}{2} \int\left|\nabla H_{t}\right|^{2} \mathrm{~d} x \leq \varepsilon_{2}\|\nabla \dot{u}\|_{L^{2}}^{2}+C\left(\varepsilon_{2}\right)\left(\left\|H_{t}\right\|_{L^{2}}^{2}+\|\nabla u\|_{L^{4}}^{2}\right) .
$$

Then, combining (2.35) and (2.37), using Young's inequality, and choosing $\varepsilon_{2}>0$ suitably small yield that

$$
\begin{aligned}
& \frac{\mathrm{d}}{\mathrm{d} t}\left(\|\sqrt{\rho} \dot{u}\|_{L^{2}}^{2}+\|\sqrt{\rho} \dot{w}\|_{L^{2}}^{2}+\left\|H_{t}\right\|_{L^{2}}^{2}\right) \\
& \quad+\left(\|\nabla \dot{u}\|_{L^{2}}^{2}+\|\nabla \dot{w}\|_{L^{2}}^{2}+\left\|\nabla H_{t}\right\|_{L^{2}}^{2}+\|2 \dot{w}-\nabla \times \dot{u}\|_{L^{2}}^{2}\right) \\
& \leq C\left(1+\|\nabla u\|_{L^{6}}^{3}+\|\nabla w\|_{L^{6}}^{3}+\left\|H_{t}\right\|_{L^{2}}^{2}+\|\nabla H\|_{H^{1}}^{2}\right) .
\end{aligned}
$$


Firstly, we use (2.4)-(2.6), (2.9), (2.10), (2.11), (2.1) and (2.2) to infer from the standard $L^{p}$-estimate that

$$
\begin{aligned}
\|\nabla u\|_{L^{6}}+\|\nabla w\|_{L^{6}} & \leq C\left(\|\operatorname{div} u\|_{L^{6}}+\|\nabla \times u\|_{L^{6}}+\|\operatorname{div} w\|_{L^{6}}+\|\nabla \times w\|_{L^{6}}\right) \\
& \leq C\left(1+\left\|G_{1}\right\|_{L^{6}}+\left\|G_{2}\right\|_{L^{6}}+\left\|V_{1}\right\|_{L^{6}}+\left\|V_{2}\right\|_{L^{6}}+\left\||H|^{2}\right\|_{L^{6}}\right) \\
& \leq C\left(1+\left\|\nabla G_{1}\right\|_{L^{2}}+\left\|\nabla G_{2}\right\|_{L^{2}}+\left\|\nabla V_{1}\right\|_{L^{2}}+\left\|\nabla V_{2}\right\|_{L^{2}}\right) \\
& \leq C\left(1+\|\sqrt{\rho} \dot{u}\|_{L^{2}}+\|\sqrt{\rho} \dot{w}\|_{L^{2}}+\|\nabla H\|_{H^{1}}\right),
\end{aligned}
$$

and

$$
\begin{aligned}
& \|\sqrt{\rho} \dot{u}\|_{L^{2}}+\|\sqrt{\rho} \dot{w}\|_{L^{2}} \\
& \leq C\left(\left\|\sqrt{\rho} u_{t}\right\|_{L^{2}}+\left\|\sqrt{\rho} w_{t}\right\|_{L^{2}}\right)+C\left(\|\sqrt{\rho} u \cdot \nabla u\|_{L^{2}}+\|\sqrt{\rho} w \cdot \nabla w\|_{L^{2}}\right) \\
& \leq C\left(\left\|\sqrt{\rho} u_{t}\right\|_{L^{2}}+\left\|\sqrt{\rho} w_{t}\right\|_{L^{2}}\right)+C\left(\|u\|_{L^{\infty}}\|\nabla u\|_{L^{2}}+\|w\|_{L^{\infty}}\|\nabla w\|_{L^{2}}\right) \\
& \leq C\left(\left\|\sqrt{\rho} u_{t}\right\|_{L^{2}}+\left\|\sqrt{\rho} w_{t}\right\|_{L^{2}}\right)+C\left(\|\nabla u\|_{L^{6}}^{\frac{1}{2}}+\|\nabla w\|_{L^{6}}^{\frac{1}{2}}\right) \text {. }
\end{aligned}
$$

Moreover, by the standard $L^{2}$-estimate of an elliptic system, we infer from $(1.1)_{4},(2.1)$, (2.2) and (2.11) that

$$
\begin{aligned}
\|\nabla H\|_{H^{1}} & \leq C\left(\left\|H_{t}\right\|_{L^{2}}+\|u \nabla H\|_{L^{2}}+\|H \nabla u\|_{L^{2}}+\|\nabla u\|_{L^{2}}\right) \\
& \leq C\left(\left\|H_{t}\right\|_{L^{2}}+\|u\|_{L^{6}}\|\nabla H\|_{L^{3}}+\|H\|_{L^{\infty}}\|\nabla u\|_{L^{2}}+1\right) \\
& \leq C\left(\left\|H_{t}\right\|_{L^{2}}+\|\nabla H\|_{L^{2}}^{\frac{1}{2}}\|\nabla H\|_{L^{6}}^{\frac{1}{2}}+1\right) \\
& \leq C\left(\left\|H_{t}\right\|_{L^{2}}+\|\nabla H\|_{H^{1}}^{\frac{1}{2}}+1\right),
\end{aligned}
$$

and hence,

$$
\|\nabla H\|_{H^{1}} \leq C\left(\left\|H_{t}\right\|_{L^{2}}+1\right) .
$$

Combining (2.39)-(2.41), we obtain

$$
\|\nabla u\|_{L^{6}}+\|\nabla w\|_{L^{6}} \leq C\left(1+\left\|\sqrt{\rho} u_{t}\right\|_{L^{2}}+\left\|\sqrt{\rho} w_{t}\right\|_{L^{2}}+\left\|H_{t}\right\|_{L^{2}}\right) .
$$

Now, putting (2.41) and (2.42) into (2.38), one has

$$
\begin{aligned}
& \frac{\mathrm{d}}{\mathrm{d} t}\left(\|\sqrt{\rho} \dot{u}\|_{L^{2}}^{2}+\|\sqrt{\rho} \dot{w}\|_{L^{2}}^{2}+\left\|H_{t}\right\|_{L^{2}}^{2}\right) \\
& \quad+\left(\|\nabla \dot{u}\|_{L^{2}}^{2}+\|\nabla \dot{w}\|_{L^{2}}^{2}+\left\|\nabla H_{t}\right\|_{L^{2}}^{2}+\|2 \dot{w}-\nabla \times \dot{u}\|_{L^{2}}^{2}\right) \\
& \leq C\left(1+\|\sqrt{\rho} \dot{u}\|_{L^{2}}^{2}+\|\sqrt{\rho} \dot{w}\|_{L^{2}}^{2}+\left\|H_{t}\right\|_{L^{2}}^{2}\right)\left(1+\left\|\sqrt{\rho} u_{t}\right\|_{L^{2}}+\left\|\sqrt{\rho} w_{t}\right\|_{L^{2}}\right),
\end{aligned}
$$

from which and (2.11), we immediately obtain (2.25) by Gronwall's inequality, (1.6) and (1.7). As a result of (2.41), we can also deduce the boundedness of $\|\nabla H\|_{H^{1}}$.

The next lemma is used to bound the density gradient and $\|\nabla u\|_{L^{1}\left(0, T ; L^{\infty}\right)}$. 
Lemma 2.8 Under the condition (2.4), it holds that for any $0 \leq T<T^{*}$,

$$
\begin{aligned}
& \sup _{0 \leq t \leq T}\left(\|\nabla \rho\|_{L^{2} \cap L^{q}}+\|\nabla u\|_{H^{1}}+\|\nabla w\|_{H^{1}}\right) \\
& \quad+\int_{0}^{T}\left(\|\nabla u\|_{L^{\infty}}+\|\nabla u\|_{W^{1, q}}^{2}+\|\nabla w\|_{W^{1, q}}^{2}\right) \mathrm{d} t \leq C
\end{aligned}
$$

for any $q \in(3,6]$.

Proof Differentiating (1.1) with respect to $x_{i}$ and multiplying it by $\left|\partial_{i} \rho\right|^{q-2} \partial_{i} \rho(q \geq 2)$ in $L^{2}$, we obtain, after integrating by parts and summing up, that

$$
\begin{aligned}
\frac{\mathrm{d}}{\mathrm{d} t} \int|\nabla \rho|^{q} \mathrm{~d} x & \leq C(q) \int\left(|\nabla u \| \nabla \rho|^{q}+\rho|\nabla \rho|^{q-1}|\nabla \operatorname{div} u|\right) \mathrm{d} x \\
& \leq C(q)\left(\|\nabla u\|_{L^{\infty}}\|\nabla \rho\|_{L^{q}}^{q}+\|\nabla \operatorname{div} u\|_{L^{q}}\|\nabla \rho\|_{L^{q}}^{q-1}\right) .
\end{aligned}
$$

It follows from (2.1), (2.4), (2.6)-(2.9), (2.25) and the interpolation inequality that for any $q \in(3,6]$,

$$
\begin{aligned}
\|\nabla \operatorname{div} u\|_{L^{q}} & \leq C\left(\left\|\nabla G_{1}\right\|_{L^{q}}+\|\nabla P\|_{L^{q}}+\|H\|_{L^{\infty}}\|\nabla H\|_{L^{q}}\right) \\
& \leq C\left(1+\left\|\nabla G_{1}\right\|_{L^{2}}+\left\|\nabla G_{1}\right\|_{L^{6}}+\|\nabla \rho\|_{L^{q}}\right) \\
& \leq C\left(1+\|\nabla \dot{u}\|_{L^{2}}+\|\nabla \dot{w}\|_{L^{2}}+\|\nabla \rho\|_{L^{q}}\right),
\end{aligned}
$$

where (2.2) and (2.25) were also used to get that $\|H\|_{L^{\infty}} \leq C$. So, putting this into (2.44) yields

$$
\frac{\mathrm{d}}{\mathrm{d} t}\|\nabla \rho\|_{L^{q}} \leq C\left(\|\nabla u\|_{L^{\infty}}+1\right)\|\nabla \rho\|_{L^{q}}+C\left(1+\|\nabla \dot{u}\|_{L^{2}}+\|\nabla \dot{w}\|_{L^{2}}\right) .
$$

We now estimate $\|\nabla u\|_{L^{\infty}}$. To do this, we first observe that

$$
(\mu+\xi) \Delta u+(\mu+\lambda-\xi) \nabla \operatorname{div} u=\rho \dot{u}+\nabla P-2 \xi \nabla \times w+(\nabla \times H) \times H .
$$

Hence, using the standard $L^{p}$-estimate of an elliptic system leads to

$$
\begin{aligned}
\left\|\nabla^{2} u\right\|_{L^{q}} & \leq C\left(\|\rho \dot{u}\|_{L^{q}}+\|\nabla P\|_{L^{q}}+\|\nabla w\|_{L^{q}}+\|H \nabla H\|_{L^{q}}\right) \\
& \leq C\left(1+\|\nabla \dot{u}\|_{L^{2}}+\|\nabla \rho\|_{L^{q}}+\|\nabla w\|_{H^{1}}\right) .
\end{aligned}
$$

From $(1.1)_{3}$ and the standard $L^{2}$-estimate of the elliptic system, we have that

$$
\|\nabla w\|_{H^{1}} \leq C\left(\|w\|_{H^{1}}+\|\sqrt{\rho} \dot{w}\|_{L^{2}}+\|\nabla u\|_{L^{2}}\right) \leq C,
$$

and then

$$
\left\|\nabla^{2} u\right\|_{L^{q}} \leq C\left(1+\|\nabla \dot{u}\|_{L^{2}}+\|\nabla \rho\|_{L^{q}}\right) .
$$


This, together with Lemmas 2.2 and 2.6, gives

$$
\begin{aligned}
\|\nabla u\|_{L^{\infty}} \leq & C\left(\|\nabla u\|_{L^{2}}+1\right)+C\left(\|\operatorname{div} u\|_{L^{\infty}}+\|\nabla \times u\|_{L^{\infty}}\right) \log \left(e+\left\|\nabla^{2} u\right\|_{L^{q}}\right) \\
\leq & C+C\left(\|\operatorname{div} u\|_{L^{\infty}}+\|\nabla \times u\|_{L^{\infty}}\right) \log \left(e+\|\nabla \dot{u}\|_{L^{2}}\right) \\
& +C\left(\|\operatorname{div} u\|_{L^{\infty}}+\|\nabla \times u\|_{L^{\infty}}\right) \log \left(e+\|\nabla \rho\|_{L^{q}}\right) .
\end{aligned}
$$

Now, if we set $f(t)=e+\|\nabla \rho\|_{L^{q}}$ and let

$$
g(t)=\left(1+\|\operatorname{div} u\|_{L^{\infty}}+\|\nabla \times u\|_{L^{\infty}}+\|\nabla \dot{u}\|_{L^{2}}+\|\nabla \dot{w}\|_{L^{2}}\right) \log \left(e+\|\nabla \dot{u}\|_{L^{2}}\right),
$$

then it is seen from $(2.45)$ and (2.48) that

$$
f^{\prime}(t)=C g(t) f(t)+C g(t) f(t) \ln f(t)
$$

due to $f(t)>1$. Thus,

$$
(\ln f(t))^{\prime} \leq C g(t)+C g(t) \ln f(t) .
$$

On the other hand, since

$$
g(t) \leq C\left(1+\|\operatorname{div} u\|_{L^{\infty}}^{2}+\|\nabla \times u\|_{L^{\infty}}^{2}+\|\nabla \dot{u}\|_{L^{2}}^{2}+\|\nabla \dot{w}\|_{L^{2}}\right),
$$

we thus deduce from (2.11), (2.25), (2.4), (2.8), (2.9) and (2.2) that

$$
\begin{aligned}
\int_{0}^{T} g(t) \mathrm{d} t & \leq C \int_{0}^{T}\left(1+\|\operatorname{div} u\|_{L^{\infty}}^{2}+\|\nabla \times u\|_{L^{\infty}}^{2}+\|\nabla \dot{u}\|_{L^{2}}^{2}+\|\nabla \dot{w}\|_{L^{2}}\right) \mathrm{d} t \\
& \leq C+C \int_{0}^{T}\left(\|\operatorname{div} u\|_{L^{\infty}}^{2}+\|\nabla \times u\|_{L^{\infty}}^{2}\right) \mathrm{d} t \\
& \leq C+C \int_{0}^{T}\left(\left\|G_{1}\right\|_{L^{\infty}}^{2}+\left\|V_{1}\right\|_{L^{\infty}}^{2}+\|P\|_{L^{\infty}}^{2}+\|H\|_{L^{\infty}}^{4}\right) \mathrm{d} t \\
& \leq C+C \int_{0}^{T}\left(\left\|G_{1}\right\|_{L^{2}}^{2}+\left\|\nabla G_{1}\right\|_{L^{2}}^{2}+\left\|V_{1}\right\|_{L^{2}}^{2}+\left\|\nabla V_{1}\right\|_{L^{2}}^{2}\right) \mathrm{d} t \\
& \leq C+C \int_{0}^{T}\left(\|\nabla \dot{u}\|_{L^{2}}^{2}+\|\nabla \dot{w}\|_{L^{2}}^{2}+\|\nabla H\|_{H^{1}}^{4}\right) \mathrm{d} t \leq C .
\end{aligned}
$$

As a result, it follows from (2.49) and Gronwall's inequality that

$$
f(t) \leq C \text { for any } 0 \leq t \leq T<T^{*},
$$

and consequently,

$$
\sup _{0 \leq t \leq T}\|\nabla \rho\|_{L^{q}} \leq C \quad \text { for any } q \in(3,6]
$$

From this and (2.25), (2.48), (2.50), one obtains

$$
\int_{0}^{T}\|\nabla u\|_{L^{\infty}} \mathrm{d} t \leq C .
$$


Taking $q=2$ in (2.45), we get, by using (2.52) and (2.25) and Gronwall's inequality, that

$$
\sup _{0 \leq t \leq T}\|\nabla \rho\|_{L^{2}} \leq C
$$

Moreover, the standard $L^{2}$-estimate of an elliptic system and $(1.1)_{2}$, together with (2.4), (2.11) and (2.25), implies

$$
\begin{aligned}
\left\|\nabla^{2} u\right\|_{L^{2}} & \leq C\left(\|\rho \dot{u}\|_{L^{2}}+\|\nabla P\|_{L^{2}}+\|H \nabla H\|_{L^{2}}+\|\nabla u\|_{L^{2}}+\|\nabla w\|_{L^{2}}\right) \\
& \leq C\left(1+\|\nabla \rho\|_{L^{2}}\right) \leq C .
\end{aligned}
$$

Similar to the proof of (2.47), there are

$$
\begin{aligned}
\left\|\nabla^{2} u\right\|_{L^{q}}+\left\|\nabla^{2} w\right\|_{L^{q}} & \leq C\left(1+\|\nabla \dot{u}\|_{L^{2}}+\|\rho \dot{w}\|_{L^{q}}+\|\nabla u\|_{L^{q}}+\|w\|_{L^{q}}\right) \\
& \leq C\left(1+\|\nabla \dot{u}\|_{L^{2}}+\|\nabla \dot{w}\|_{L^{2}}\right), \quad \forall q \in(3,6],
\end{aligned}
$$

where we have used (2.1), (2.11), (2.46), (2.51) and (2.54). From this, together with (2.25), (2.46) and (2.51)-(2.54), we can deduce (2.43).

As a consequence of Lemmas 2.6-2.8, we have the following lemma.

Lemma 2.9 Under the condition (2.4), it holds that for any $0 \leq T<T^{*}$,

$$
\sup _{0 \leq t \leq T}\left(\left\|\sqrt{\rho} u_{t}\right\|_{L^{2}}^{2}+\left\|\sqrt{\rho} w_{t}\right\|_{L^{2}}^{2}\right)+\int_{0}^{T}\left(\left\|\nabla u_{t}\right\|_{L^{2}}^{2}+\left\|\nabla w_{t}\right\|_{L^{2}}^{2}\right) \mathrm{d} t \leq C
$$

The proof is the same as that of Lemma 3.6 in [20] and is omitted here.

With the help of Lemmas 2.3, 2.6-2.9 and the local existence theorem, we can complete the proof of Theorem 1.2 by the contradiction arguments. In fact, in view of Lemmas 2.3, 2.6-2.9, it is easy to see that the functions $(\rho, u, w, H)\left(x, T^{*}\right)=\lim _{t \rightarrow T^{*}}(\rho, u, w, H)$ have the same regularities imposed on the initial data (1.5) at the time $t=T^{*}$. This implies that the compatibility conditions (1.6) and (1.7) are satisfied at the time $T^{*}$. Thus, we can take $(\rho, u, w, H)\left(x, T^{*}\right)$ as the initial data and apply the local existence theorem to extend the local strong solutions beyond $T^{*}$. This contradicts the assumption that $T^{*}$ is the maximal time of existence.

Competing interests

The author declares that they have no competing interests.

\section{Acknowledgements}

This work is partially supported by the Fundamental Research Funds for the Central Universities (Grant No. 11QZR16), the National Natural Science Foundation of China (Grant No. 11001090).

Received: 18 February 2013 Accepted: 16 June 2013 Published: 1 July 2013

\section{References}

1. Berkovski, B, Bashtovoy, V: Magnetic Fluids and Applications Handbook. Begell House, New York (1996)

2. Hatzikonstantinou, PM, Vafeas, P: A general theoretical model for the magnetohydrodynamic flow of micropolar magnetic fluids. Application to Stokes flow. Math. Methods Appl. Sci. 33, 233-248 (2009)

3. Papadopoulos, PK, Vafeas, P, Hatzikonstantinou, PM: Ferrofluid pipe flow under the influence of the magnetic field of a cylindrical coil. Phys. Fluids 24, 122002 (2012). doi:10.1063/1.4769177

4. Rosensweig, RE: Ferrohydrodynamics. Dover Publications, New York (1997) 
5. Rojas-Medar, MA: Magneto-micropolar fluid motion: existence and uniqueness of strong solution. Math. Nachr. 188 301-319 (1997)

6. Ortega-Torres, EE, Rojas-Medar, MA: Magneto-micropolar fluid motion: global existence of strong solutions. Abstr. Appl. Anal. 4, 109-125 (1999)

7. Yuan, J: Existence theorem and blow-up criterion of strong solutions to the magneto-micropolar fluid equations. Math. Methods Appl. Sci. 31, 1113-1130 (2008)

8. Yuan, BQ: Regularity of weak solutions to magneto-micropolar fluid equations. Acta Math. Sci. 30, 1469-1480 (2010)

9. Gala, S: Regularity criterion for 3D magneto-micropolar fluid equations in the Morrey-Campanato space. Nonlinear Differ. Equ. Appl. 17, 181-194 (2010)

10. Volpert, Al, Khudiaev, SI: On the Cauchy problem for composite system of nonlinear equations. Mat. Sb. 87, 504-528 (1972)

11. Fan, JS, Yu, WH: Strong solution to the compressible MHD equations with vacuum. Nonlinear Anal., Real World Appl. 10, 392-409 (2009)

12. $\mathrm{Xu}, \mathrm{XY}$, Zhang, JW: A blow-up criterion for 3D compressible magnetohydrodynamic equations with vacuum. Math. Models Methods Appl. Sci. 22, 1150010 (2012)

13. Mujakovic, N: One-dimensional flow of a compressible viscous micropolar fluid: a local existence theorem. Glas. Mat. 33(53), 71-91 (1998)

14. Mujakovic, N: One-dimensional flow of a compressible viscous micropolar fluid: a global existence theorem. Glas. Mat. 33(53), 199-208 (1998)

15. Chen, MT: Global strong solutions for the viscous, micropolar, compressible flow. J. Partial Differ. Equ. 24, 158-164 (2011)

16. Amirat, $\mathrm{Y}$, Hamdache, $\mathrm{K}$ : Weak solutions to the equations of motion for compressible magnetic fluids. J. Math. Pures Appl. 91, 433-467 (2009)

17. Lions, PL: Mathematical Topics Influid Mechanics. Vol. 2. Compressible Models. Oxford University Press, New York (1998)

18. Feireisl, E, Novotny, A, Petzeltová, H: On the existence of globally defined weak solutions to the Navier-Stokes equations. J. Math. Fluid Mech. 3, 358-392 (2001)

19. Chen, MT: Unique solvability of compressible micropolar viscous fluids. Bound. Value Probl. 2012, 32 (2012)

20. Chen, MT, Huang, B, Zhang, JW: Blowup criterion for three-dimensional equations of compressible viscous micropolar fluids with vacuum. Nonlinear Anal. 79, 1-11 (2013)

21. Huang, XD, Li, J, Xin, ZP: Serrin-type criterion for the three-dimensional viscous compressible flows. SIAM J. Math. Anal. 43(4), 1872-1886 (2011)

22. Choe, JH, Kim, H: Strong solutions of the Navier-Stokes equations for isentropic compressible fluids. J. Differ. Equ. 190 504-523 (2003)

doi:10.1186/1687-2770-2013-160

Cite this article as: Zhang: Blow-up criterion for 3D compressible viscous magneto-micropolar fluids with initial vacuum. Boundary Value Problems 2013 2013:160

\section{Submit your manuscript to a SpringerOpen ${ }^{\circ}$ journal and benefit from:}

- Convenient online submission

Rigorous peer review

- Immediate publication on acceptance

- Open access: articles freely available online

- High visibility within the field

- Retaining the copyright to your article 